\title{
Lesões por Esforços Repetitivos e Sofrimento Mental em Diferentes Profissões
}

Maria Engrácia de Carvalho

Chaves'

Carlos Antônio Melgaço

Valadares $^{2}$

Maria Lúcia Maia Ribeiro Lino ${ }^{3}$

Marco Bussacos ${ }^{3}$

\author{
Repetitive Strain Injuries (RSI) and \\ Mental Suffering in Different \\ Professions
}

'FUNDACENTRO - Ministério do Trabalho e Emprego.

${ }^{2}$ Sindicato dos Bancários da Bahia. Participação : Comissão Intersindical de LER.

${ }^{3}$ Trabalho apresentado no XVlth World Congress on Safety and Health at Work, na Áustria , no período de 26 a 31 de maio de 2002
Estudo que tem como objetivo específico comparar os resultados de um indicador de sofrimento mental em portadores de LER/DORT, de acordo com diferentes profissões. $O$ instrumento central da pesquisa foi um questionário auto - aplicado contendo questões objetivas e subjetivas, com 120 perguntas. O estado de saúde mental foi avaliado através da inclusão dos ítens do SRQ-20 (Self-Report Questionnaire). Os testes estatísticos utilizados foram : Cochran Armitage, qui-quadrado e t-student. Os principais resultados foram: apenas os trabalhadores de telecomunicações e processamento de dados (TELEPROCESS), trabalhavam, em sua maioria, no horário de turnos. A grande maioria dos trabalhadores de todas as profissões referiam realizar horas-extra, com exceção dos trabalhadores de TELEPROCESS $(33,33 \%)$. A maioria dos trabalhadores de todos os setores, com exceção do setor TELEPROCESS, queixou-se de pressão por parte das chefias. A percentagem de positividade do SRQ-20 foi de aproximadamente $90 \%$ - não houve diferença estatisticamente significante em relação às profissões. Chegamos à conclusão que o adoecimento por LER interfere nos níveis de sofrimento mental , aumentando-o homogeneamente entre as diferentes profissões.

Palavras-chaves Lesões por Esforços Repetitivos, Distúrbios Osteomusculares Relacionados ao Trabalho, Sofrimento Mental, Organização do Trabalho.

The objective was to compare one indicator of mental suffering among workers affected by repetition strain injuries (RSI), according different professions. The main instrument for the study was a broad questionnaire consisting of 120 objective and subjective questions, including SRQ-20 (Self Report Questionnaire). The utilized statistical tests were : chi-square, Cochran Armitage and t-student. The main results were: only the telecommunication workers worked in shift work, in majority. The great majority of all the professions, except telecommunication workers, refered overtime work performed. The majority of all the sectors, except telecommunication, refered bosses' pressure. The SRQ-20 positivity frequence was $90 \%$, aproximately. There were not statistically significant differences between the SRQ-20 results in relationship to the professions. The high and similar SRQ-20 positivity frequencies point out the relevance of being a population with established diagnosis of RSI, what increases the mental suffering levels among the different professions.

Keywords Repetition Strain Injuries (RSI), Mental Suffering, Work Organization. 


\section{Apresentação}

Em 1997, iniciou-se um amplo estudo epidemiológico descritivo com 105 portadores de Lesões por Esforços Repetitivos (LER) / Distúrbios Osteomusculares Relacionados ao Trabalho (DORT), por solicitação da Comissão Intersindical de LER/BA. Este estudo foi realizado através de um trabalho conjunto da FUNDACENTRO/CRBA, do Sindicato dos Bancários da Bahia e da referida Comissão.

O objetivo geral de toda a pesquisa foi desenvolver amplo estudo com portadores de LER, buscando-se compreender melhor o processo de adoecimento e a interferência da doença na própria vida das pessoas acometidas (CHAVES e cols., no prelo). A partir desse trabalho inicial, foram desdobrados outros, inclusive o presente estudo.

\section{Introdução}

Para se entender as mudanças que se processam no mundo do trabalho há que se pensar a atendências da economia caracterizada pela chamada globalização, com reestruturação e intensa competição entre empresas, terceirização com precarização do trabalho e a ofensiva contra direitos históricos conquistados pelos trabalhadores, em nome da flexibilização.

Na tentativa de avaliar o impacto das mudanças no mundo do trabalho sobre a saúde dos trabalhadores, alguns estudos têm partido do conjunto corpo-mente, buscando entender como se relacionam as lesões físicas e o sofrimento mental.

BORGES (1999), estudando as relações entre trabalho bancário e adoecimento físico e mental, destaca a presença e associação entre distúrbios psicoemocionais e osteomusculares. Os primeiros estariam associados, em seu estudo, a fatores da organização do trabalho, tais como: sobrecarga de trabalho, controle de chefia e sentimentos de desvalorização.

Analisando a diferença dos índices da freqüência de distúrbios mentais menores, que foram maiores, com o de LER, BORGES(1999) levanta duas possibilidades: a primeira, que o sofrimento psíquico poderia ser indicador precoce das LER; e a segunda, que nem todo sofrimento psíquico estaria relacionado às LER e, na ausência de algumas condições de sobrecarga osteomuscular, encontraria outra forma de expressão mórbida.

No que se refere ao atendimento clínico no ambulatório de Saúde Mental do CRST André Gabois, ROCHA e cols (2001) mencionam a presença de trabalhadores com LER/DORT e quadros de depressão, entre os pacientes que procuraram o Serviço.

Em publicação do Ministério da Saúde (2001), embora seja feita a ressalva de que não se pode generalizar a indicação de psicoterapia para todos os portadores de LER, já que a indicação deverá ser feita a partir da avaliação de cada caso em particular e do desejo de cada um, a observação feita é que "em algum momento da evolução de LER/DORT, o paciente tem necessidade de apoio psicológico por parte de um profissional", segundo a experiência dos autores.

A experiência de dois anos de atendimento de portadores de LER em um trabalho de psicoterapia de grupo possibilita-nos observar a importância de alguns fatores na determinação das LER, especialmente a pressão por parte das chefias, levando a uma tensão emocional claramente relacionada ao adoecimento; por outro lado, o sofrimento mental acarretado pela presença do adoecimento tem estado presente durante todo o período de atendimento, o que coloca a necessidade de reflexão sobre dificuldades e possibilidades no redirecionamento da vida, buscando-se alternativas para a realidade limitante das LER (CHAVES, 2002).

$O$ sofrimento mental vem sendo estudado em "trabalhadores sadios" de diversas categorias, tais como bancários, telecomunicações, processamento de dados, siderúrgicos e muitas outras (BORGES, 1990; BORGES, 1999; CHAVES e cols, 1998; FERNANDES, 1992; JARDIM, 1994; PITTA, 1990; SILVA FILHO e cols, 1993). Nestes estudos citados, o instrumento utilizado como indicador de distúrbios mentais menores foi - SRQ-20, cujos índices variaram de 20 a $37,7 \%$ de positividade.

No presente estudo, pretendemos conhecer em que magnitude ocorre o sofrimento mental em portadores de LER e se há variações de intensidade deste sofrimento de 
acordo com as diversas profissões estudadas.

\section{Materiais e métodos}

Foi desenvolvido um estudo epidemiológico descritivo (corte transversal). A população de estudo constituiu-se, inicialmente, de 105 portadores de Distúrbios Osteomusculares Relacionados ao Trabalho que frequentaram as reuniões mensais da Comissão Intersindical de LER/DORT no CESAT(Centro de Estudos da Saúde dos Trabalhadores), no período de julho a setembro de 1999. Os trabalhadores pertenciam às categorias de bancários, comerciários, processamento de dados, telecomunicações e outras. Um participante da pesquisa foi excluído, pois omitiu a informação sobre a que categoria pertencia.

O instrumento central da pesquisa foi um questionário auto- aplicado, contendo questões objetivas e subjetivas, com 120 perguntas. $O$ estado de saúde mental foi avaliado através do SRQ-20 (Self-Report Questionnaire). Este questionário, contendo 20 questões, foi desenvolvido pela OMS com - objetivo de rastrear distúrbios psiquiátricos menores, na população que se dirigia a ins- tituições de cuidados primários de saúde, em países em desenvolvimento (MARI \& WILLIAMS, 1986). O SRQ-20 foi considerado positivo com um ponto de corte de 8 para mulheres e 6 para homens.

Houve, inicialmente, um estudo piloto, que favoreceu a escolha da população de estudo definitiva. Toda a equipe foi treinada para a coleta de dados. Durante a coleta, houve reuniões semanais para discussão das dúvidas.

Os testes estatísticos utilizados foram : Cochran-Armitasge, Qui-quadrado e $t$-Student.

\section{Resultados/discussão}

A média de idade dos trabalhadores foi de 41 anos, aproximadamente. Cerca de $87 \%$ da população eram mulheres e $12,4 \%$, homens. Uma pessoa não informou o sexo.

Na tabela 1, observa-se que apenas os trabalhadores da área de Telecomunicações e Processamento de Dados (TELE/ PROCESS) trabalham, predominantemente, em turno (57.14\%). Seguem-se os comerciários (33.3\%), bancários (23.5\%) e outras profissões (13.64\%). Essas diferenças foram, estatisticamente, significantes.

Tabela 1 Distribuição dos trabalhadores com LER, de acordo com área de trabalho e sistema de horários $c^{2}=10,81 ; p<0.05$.

\begin{tabular}{|c|c|c|c|c|}
\hline \multirow{2}{*}{$\begin{array}{l}\text { Área } \\
\text { de } \\
\text { Trabalho }\end{array}$} & \multirow{2}{*}{$\begin{array}{l}\text { Horário } \\
\text { de } \\
\text { Trabalho }\end{array}$} & Administrativo & Turnos & Total \\
\hline & & N $\quad \%$ & $\mathbf{N} \quad \%$ & $\mathbf{N} \quad \%$ \\
\hline Banco & & $26(76.47)$ & $8(23.53)$ & $34(32,69)$ \\
\hline Comércio & & $18(66.67)$ & $9(33.33)$ & $27(25,96)$ \\
\hline Tele/Proce & ess & $9(42.86)$ & $12(57.14)$ & $21(20,19)$ \\
\hline Outros & & $19(86.36)$ & $3(13.64)$ & $22(21,16)$ \\
\hline Total & & $72(69.23)$ & $32(30.77)$ & $104(100,00)$ \\
\hline
\end{tabular}


Na tabela 2, observa-se que todas as profissões realizam horas-extras, destacandose os comerciários $(96.30 \%)$, seguidos dos bancários (91.18\%), outras profissões (86.36\%) e TELE/PROCESS(66.67\%). O destaque maior diz respeito ao menor percentual de realização de horas-extras no segmento TELE/PROCESS. Resultado esperado, uma vez que há um controle maior na área de processamento de dados, no que se refere ao exercício de horas-extras, com legislação mais clara e objetiva em relação ao número de horas permitidas, pelo menos, para os que trabalham em terminais de computador. As diferenças encontradas não foram, estatisticamente, significantes.

Tabela 2 Distribuição dos Trabalhadores com LER, de acordo com área de trabalho e realização de horas - extras Cochran-Armitage $Z=1,14 ; p=0,2533$.

\begin{tabular}{|c|c|c|c|c|c|c|c|c|c|}
\hline \multirow{2}{*}{$\begin{array}{l}\text { Área } \\
\text { de } \\
\text { trabalho }\end{array}$} & \multirow{2}{*}{$\begin{array}{l}\text { Realização } \\
\text { de horas- } \\
\text { extras }\end{array}$} & \multicolumn{2}{|c|}{ Sim } & \multicolumn{2}{|c|}{ Não } & \multicolumn{2}{|c|}{ Sem dado } & \multicolumn{2}{|c|}{ Total } \\
\hline & & $\mathbf{N}$ & $\%$ & $\mathbf{N}$ & $\%$ & $\mathbf{N}$ & $\%$ & $\mathbf{N}$ & $\%$ \\
\hline \multicolumn{2}{|l|}{ Banco } & & $(91,18)$ & 3 & $(8,82)$ & & $(0,00)$ & 34 & $(32,69)$ \\
\hline \multicolumn{2}{|c|}{ Comércio } & & $(96,30)$ & 1 & $(3,70)$ & 0 & $(0,00)$ & 27 & $(25,96)$ \\
\hline \multicolumn{2}{|c|}{ Tele/Process } & & $(66,67)$ & & 33,33) & 0 & $(0,00)$ & 21 & $(20,19)$ \\
\hline \multicolumn{2}{|l|}{ Outras } & & $(86,36)$ & 2 & $(9,09)$ & & $(4,55)$ & 22 & $(21,16)$ \\
\hline \multicolumn{2}{|l|}{ Total } & & $(86,54)$ & 13 & 12,50) & & $(0,96)$ & 104 & $100,00)$ \\
\hline
\end{tabular}

A profissão que mais se destaca na queixa relacionada à pressão de chefia é a de bancários (82.35\%) - tabela 3. Em estudo com caixas bancários, realizado por BORGES (1999), a variável "problemas com chefia" foi correlacionada, tanto aos distúrbios psico-emocionais, quanto às LER.

Por outro lado, o menor percentual de queixa referente a essa variável é dos trabalhadores da área de TELE/PROCESS (33.33\%). Este dado está de acordo com algumas discussões sobre a ausência de uma chefia constante e vigilante no trabatho de turno, como ocorre no turno administrativo. Essas diferenças foram, estatisticamente, significantes.

Tabela 3 Distribuição dos Trabalhadores com LER, de acordo com área de trabalho e queixas acerca da pressão das chefias $c^{2}=14,05 ; p<0,05$.

\begin{tabular}{|c|c|c|c|c|c|c|c|c|}
\hline \multirow{2}{*}{$\begin{array}{l}\text { Área } \\
\text { de } \\
\text { trabalho }\end{array}$} & \multirow{2}{*}{$\begin{array}{l}\text { Pressão } \\
\text { de } \\
\text { Chefias }\end{array}$} & \multicolumn{2}{|c|}{$\operatorname{Sim}$} & \multicolumn{2}{|c|}{ Não } & Sem dado & \multicolumn{2}{|c|}{ Total } \\
\hline & & $\mathrm{N}$ & $\%$ & $\mathbf{N}$ & $\%$ & N $\%$ & $\mathbf{N}$ & $\%$ \\
\hline \multicolumn{2}{|l|}{ Banco } & \multicolumn{2}{|c|}{$28(82,35)$} & \multicolumn{2}{|c|}{$6(17,65)$} & $0(0,00)$ & 34 & $(32,69)$ \\
\hline \multicolumn{2}{|l|}{ Comércio } & \multicolumn{2}{|c|}{$18(66,67)$} & \multicolumn{2}{|c|}{$9(33,33)$} & $0(0,00)$ & & $(25,96)$ \\
\hline \multicolumn{2}{|c|}{ Tele/Process } & \multicolumn{2}{|c|}{$7(33,33)$} & \multicolumn{2}{|c|}{$14(66,67)$} & $0(0,00)$ & & $(20,19)$ \\
\hline \multicolumn{2}{|l|}{ Outras } & \multicolumn{2}{|c|}{$11(50,00)$} & \multicolumn{2}{|c|}{$9(40,91)$} & $2(9,09)$ & & $(21,16)$ \\
\hline \multicolumn{2}{|l|}{ Total } & \multicolumn{2}{|c|}{$64(61,54)$} & \multicolumn{2}{|c|}{$38(36,54)$} & $2(1,92)$ & 104 & $(100,00)$ \\
\hline
\end{tabular}


Na tabela 4, observa-se a positividade do $S R Q$, segundo as profissões. Os valores de positividade foram semelhantes para os bancários (91.18\%), comerciários (92.0\%) e outras profissões (95.45\%). Os TELE/
PROCESS tiveram um índice mais baixo de positividade do SRQ (80.95\%). Os resultados não foram, estatisticamente, significantes.

Tabela 4 Distribuição dos Trabalhadores com LER, de acordo com área de trabalho e positividade do SRQ-20 *2 não responderam ao SRQ-20 Cochran-Armitage $Z=0,6933 ; p=$ 0,4881 .

\begin{tabular}{|c|c|c|c|c|}
\hline Área & Sim & Não & & otal \\
\hline $\begin{array}{l}\text { de } \\
\text { trabalho }\end{array}$ & $\mathrm{N} \quad \%$ & N $\%$ & $\mathbf{N}$ & $\%$ \\
\hline Banco & $31(91,18)$ & $3(8,82)$ & 34 & $(33,33)$ \\
\hline Comércio & $23(92,00)$ & $2(8,00)$ & 25 & $(24,51)$ \\
\hline Tele/Process & $17(80,95)$ & $4(19,05)$ & 21 & $(20,59)$ \\
\hline Outras & $21(95,45)$ & $1(4,55)$ & 22 & $(21,57)$ \\
\hline Total & $92(90,19)$ & $10(9,80)$ & $102^{*}$ & $(100,00)$ \\
\hline
\end{tabular}

Em resultados referentes a estudos com "trabalhadores sadios", foram encontradas as seguintes frequências de SRQ+ : entre dois grupos de bancários, $25 \%$ e $23,6 \%$ (SILVA FILHO e cols, 1993); em um prédio de um banco estatal, 20,6\% (CHAVES e cols , 1998); $37.7 \%$ entre caixas de um banco estatal (BORGES, 1999); entre trabalhadores de processamento de dados de três empresas distintas, uma variação de 20 a $24 \%$ (FERNANDES, 1992); 19,4\% entre trabalhadores siderúrgicos (BORGES, 1990); em estudo conduzido por Jardim (1994) com condutores de metrô, $25,8 \%$, e, dentre trabalhadores de instituições hospitalares , 20,8\% (PITTA, 1990).

\section{Conclusão}

O primeiro ponto que chama a atenção são os elevados índices de SRQ-20+ que foram encontrados nesta população, demonstrando uma situação de sofrimento mental generalizada. Quando comparamos os nossos resultados com aqueles de outros estudos realizados com "trabalhadores-sadios", percebemos a imensa distância entre eles. Nos estudos com "trabalhadores sadios", os índices de SRQ+ variaram de $20 \%$ a $37,7 \%$, enquanto que o índice ge- ral de SRQ+ nas diversas profissões estudadas foi de $90 \%$. Cremos que essa diferença deve-se ao fato de tratar-se de uma população enferma, o que interfere no nível de sofrimento mental.

Apesar das diferenças encontradas entre as profissões referentes a alguns fatores da organização do trabalho, não houve diferença, estatisticamente, significante, entre os índices de SRQ+. Esta homogeneidade nos índices das profissões estudadas reforça a idéia de que os valores elevados devem-se mais à condição do adoecimento do que à diversidade das condições de trabalho nas categorias estudadas, apontando para uma forte associação entre LER e sofrimento mental. A doença chegou para todos e com ela o sofrimento mental. O que os iguala é a condição limitante do adoecimento, que traz consigo, repercussões sérias sobre o estado emocional e psíquico dos trabalhadores. $\bigcirc$ estudo de corte transversal aponta para uma associação, mas não permite aprofundar questões sobre causalidade.

A análise inicial das perguntas subjetivas do estudo geral, apresentada em eventos por Chaves $(2000 ; 2001)$, indica a percepção unânime dos participantes do estudo de que o adoecimento por LER repercutiria sobre o estado psíquico, e a percepção 
da maioria dos trabalhadores, de que o trabalho seria desencadeador de sofrimento mental e este, por sua vez, favoreceria o adoecimento por LER. Ou seja, formas de organização do trabalho autoritárias e exigentes com a produção favorecem, tanto o sofrimento psíquico, como a ocorrência de distúrbios osteomusculares. Como refere Borges (1999), as "LER podem ser entendidas como a expressão patológica em nível físico e psíquico do conflito na organização do trabalho".

Os resultados elevados da frequência do SRQ-20+ neste estudo são alarmantes e chamam a atenção para a necessidade de uma atuação conjunta na prevenção de agravos à saúde do trabalhador, visando à proteção, tanto da saúde física, como da saúde mental.

\section{Referências Bibliográficas}

BORGES, L.H. Transtornos mentais entre trabalhadores de uma usina siderúrgica. São Paulo, 1990. (Dissertação de Mestrado, Faculdade de Medicina da Universidade de São Paulo).

BORGES, L. H. Sociabilidade, Sofrimento Psíquico e Lesões por Esforços Repetitivos em processos de trabalho repetitivos: estudo de caixas bancários. Rio de Janeiro, 1999. (Tese de Doutoramento, Instituto de Psiquiatria da Universidade Federal do Rio de Janeiro).

CHAVES, M. E. C. e cols. Organização do trabalho e saúde de bancários Salvador, Bahia. - Revista Brasileira de Saúde Ocupacional, 25 (93/94): 39 - 56, 1998.

CHAVES, M. E. C. e cols. Trabajo, L.E.R. y sufrimiento mental : el dolor del proceso del padecimiento en los datos objetivos y en la palabra del trabajador. Trabalho apresentado na III Conferencia Internacional de Psicologia de la Salud em Havana, Cuba, 27/ 11 a 01/12/2000, e no ler. Congreso Latinoamericano de Psicologia de la Salud em Veracruz, México, 21 a 26/ 05/2001.

CHAVES, M. E. C. "Psicoterapia em Grupo com portadores de LER". Relatório Parcial. FUNDACENTRO/BA, fev. 2002. 9 p.(mimeo) .
CHAVES, M. E. C. e cols. "Organização do Trabalho e efeitos sobre a saúde de trabalhadores: L.E.R. e sofrimento mental". ( Revista Brasileira de Saúde Ocupacional - no prelo).

FERNANDES, S. R. P. Trabalho informatizado e distúrbios psico emocionais - estudo seccional entre três empresas de processamento de dados em Salvador-Bahia. Salvador, 1992. (Dissertação de Mestrado, Departamento de Medicina Preventiva da Universidade Federal da Bahia).

JARDIM, S.R. Processo de trabalho e sofrimento psíquico: o caso dos pilotos do Metrô carioca. Rio de Janeiro, 1994. (Tese de Doutoramento, Instituto de Psiquiatria da Universidade Federal do Rio de Janeiro).

MARI, J.J. e WILLIAMS, P. A validity study of a psychiatric screening questionnaire (SRQ) in primary care in the city of São Paulo. British Journal of Psychiatric, 148 :23-326, 1986.

MINISTÉRIO DA SAÚDE - LER/DORT : Dilemas, Polêmicas e Dúvidas - Série A Normas e Manuais Técnicos, n. 104. Brasília, 2001. 23 p.

PITTA, A. M. F. Trabalho hospitalar e sofrimento psíquico. São Paulo, 1989. (Tese de Doutoramento, Faculdade de 
Medicina da Universidade de São Paulo) .

ROCHA,L.E.; BATISTA, M.L.; MENDONCCA, M.G.V. Saúde Mental e Trabalho : Contribuição para o Reconhecimento da Relação entre Situação de Trabalho e Distúrbios Psíquicos. Revista Brasileira de Saúde Ocupacional, 26 (99/100): 81 - 98, 2001.
SILVA FILHO, J. F. e cols. Organização do Trabalho e Saúde Mental. Estudo das relações entre a prevalência de doenças mentais e organização do trabalho bancário no município do Rio de Janeiro. Textos para discussão n.7, Fórum de Ciência e Cultura/ UFRJ. Rio de Janeiro, 1993. 